\title{
Pervasive Activity Logging for Indoor Localization in Smart Homes
}

\author{
Nirmalya Thakur* \\ Department of EECS, University of Cincinnati, Cincinnati, \\ OH 45221-0030, USA \\ thakurna@mail.uc.edu
}

\author{
Chia Y. Han \\ Department of EECS, University of Cincinnati, Cincinnati, \\ OH 45221-0030, USA \\ han@ucmail.uc.edu
}

\begin{abstract}
The scientific contribution of this work to address multiple global societal and economic challenges associated with the increasing aging population is primarily two-fold. First, it presents and discusses a new computing methodology - Pervasive Activity Logging that involves the creation of an adaptive and semantic collection or ' log' of human activities in Internet of Things (IoT)-based pervasive environments, along with the characteristics of user interactions associated with these activities in terms of the contextual, behavioral, spatial, and temporal information. Second, by using this concept of Pervasive Activity Logging, this paper presents a novel machine learning and pattern recognition-based approach for Indoor Localization during different activities performed within the premises of an IoT-based pervasive environment, such as a Smart Home. This learning approach used a Random Forest-based classification model and detected the user's indoor location with an accuracy of $83.02 \%$. These methodologies were developed by integrating the latest advancements from Pervasive Computing, Big Data, Information Retrieval, Internet of Things, Human-Computer Interaction, Machine Learning, and Pattern Recognition. The results presented and discussed uphold the relevance, potential, and importance of these methodologies for contributing towards independent living, healthy aging, and improved quality of life of elderly people in the future of IoT-based pervasive environments, such as Smart Homes.
\end{abstract}

\section{CCS CONCEPTS}

- Human-Centered Computing; - Ubiquitous and Mobile Computing; • Computing Methodologies; • Machine Learning;

\section{KEYWORDS}

pervasive computing, smart homes, activities of daily living, activity recognition, indoor localization, elderly population, big data, machine learning, internet of things

ACM Reference Format:

Nirmalya Thakur and Chia Y. Han. 2021. Pervasive Activity Logging for Indoor Localization in Smart Homes. In 2021 4th International Conference

${ }^{*}$ Corresponding author

Permission to make digital or hard copies of all or part of this work for personal or classroom use is granted without fee provided that copies are not made or distributed for profit or commercial advantage and that copies bear this notice and the full citation on the first page. Copyrights for components of this work owned by others than ACM must be honored. Abstracting with credit is permitted. To copy otherwise, or republish, to post on servers or to redistribute to lists, requires prior specific permission and/or a fee. Request permissions from permissions@acm.org.

DSIT 2021, fuly 23-25, 2021, Shanghai, China

(c) 2021 Association for Computing Machinery.

ACM ISBN 978-1-4503-9024-8/21/07 ..\$15.00

https://doi.org/10.1145/3478905.3478955 on Data Science and Information Technology (DSIT 2021), fuly 23-25, 2021, Shanghai, China. ACM, New York, NY, USA, 10 pages. https://doi.org/10. 1145/3478905.3478955

\section{INTRODUCTION}

People live longer now. The aging population of the world is increasing rapidly. Based on this rate of increase, the aging population, currently at 962 million [1], is projected to become 1.6 billion by 2050 [2]. Society has never dealt with so many and so diverse elderly people. The diversities associated with this big demographic group are due to the multiple limitations of aging, which poses a host of challenges such as cognitive impairment, behavioral disorders, motor disabilities, dementia, and visual impairments. When the decline of physical and mental abilities that elderly people face starts to affect their daily routines, caregivers' assistance becomes necessary. Thus, human resources are drawn in. These very needs are posing a huge burden on the world economy. According to World Health Organization, the global cost of looking after elderly people with Dementia was USD 818 billion in 2015, which was approximately equal to $1.1 \%$ of the global domestic product (GDP), and these costs are expected to rise steeply over the next few years [3].

On a global scale, there is a shortage of caregivers [4], and it creates two major challenges. First, caregiving costs are rising rapidly, and affording caregivers is becoming a challenge for low-income families. Second, due to the limited number of caregivers, the quality of care is getting compromised. This is because overworked and overburdened caregivers quite often do not have the patience and time to address the multiple needs of the elderly in a way that meets the highest standards of care. So, it is the need of the hour to look into solutions with pervasive computing technologies, such as the Internet of Things (IoT)-based pervasive living environments. As living infrastructure changes, we anticipate that the future will include interconnected smart cities, smart communities, and smart homes. Smart homes of the future will possess the technology to control sensors and devices for the comfort and safety of the living space and have the ability to understand the activities of the tenants of the buildings to interact with them accordingly. To perform such interactions with diverse users, the future of pervasive living spaces need to be able to sense, anticipate, adapt, predict, and respond to human behavior and the associated diverse needs and be equipped with the functionalities of a Personal Care System (PCS) that involves care anywhere, care networking, and care customization [5]. One approach to developing such functionalities in the future of smart living environments involves creating an adaptive and semantic collection of human activities in terms of the contextual, behavioral, spatial, and temporal information associated with the 
characteristics of these activities of interest. We define such a collection or 'log' of human activities as Pervasive Activity Logging. By drawing inspiration from the concept of interest-based lifelogging introduced in [6], we define Pervasive Activity Logging (PAL) as a computing methodology that can mine, study, track, analyze, and interpret the dynamic and diversified nature of both macro and micro components of user interactions of different users, during different activities performed within the confines of a pervasive environment. A pervasive computing-based environment permits the realization of a continually growing and adapting ' log,' or collection of such user interactions, along with the associated contextual and behavioral information for each of these activities to provide the basis for detection and recognition of abnormalities that render the needs for assistance. This is critical to improving the quality of life and contributing towards assisted living and healthy aging of the global elderly population.

A computing methodology, such as PAL, can have multiple applications to improve the quality of life and to contribute towards assisted living and healthy aging of the global elderly population. The study of Activities of Daily Living (ADL) and PAL requires the specification of both the temporal and spatial parameters of the activities. While the temporal components are convenient to track, the spatial information related to the human subject's motion and whereabouts in an indoor environment based on the varying individual needs, motives, and goals [7] leads to the problem of indoor localization that requires special attention. In a broad manner, an Indoor Localization System may be defined as a host of technologies, networks, and devices that communicate with each other to track and locate individuals, objects, and assets in indoor environments. Here, by indoor environments, we refer to fully or partially closed indoor settings where navigation technologies such as Global Positioning Systems (GPS) and Global Navigation Satellite Systems (GNSS) fail to work [8].

The dynamic location information provides the spatial context for the activities. Likewise, knowing the activities being carried out, the history of activities, the related behaviors, and the associated user interactions [7] can help to determine the indoor area or location where a specific activity is likely to be performed by an individual. There are multiple challenges associated with the aging population that can be readily addressed by the intersection of Indoor Localization with the PAL methodology. Some of these include - (1) detection of an elderly fall during activities and communicating the indoor location information to caregivers and healthcare providers; (2) studying human behavior for detecting instances when elderly suffering from Dementia or Alzheimer's face Freezing of Gait (FOG); (3) navigation of the visually impaired to perform their daily routine tasks independently; (4) assistance to the visually impaired in interacting with specific context parameters in pervasive settings unfamiliar to them; (5) helping elderly people with memory issues to navigate to different indoor locations for performing daily routine activities by recommending specific locations or navigation-related information; and (6) interpreting the behavior of elderly to detect and analyze sudden instances of cramps, behavioral disorders or motor impairments to automatically alert caregivers.
Because of the multiple global research challenges that a PAL methodology for Indoor Localization can address, this paper specifically focuses on the same. This paper is organized as follows. We present an overview of similar works in this field in Section 2. The methodology of Pervasive Activity Logging for Indoor Localization is further discussed in Section 3. Section 4 presents the results and findings. Conclusion and scope for future work are outlined in Section 5.

\section{LITERATURE REVIEW}

This section outlines the recent works from Pervasive Computing, Indoor Localization, and Human Activity Recognition and analysis that primarily address multiple challenges related to assisted living in Smart Homes and similar IoT-based pervasive environments.

Ranieri et al. [9] proposed an activity recognition approach based on setting up a data collection framework using a host of wearable and wireless sensors in a Smart Home environment. The system studied the data from all these sensors to analyze the user interactions to recognize human activities. Fahad et al. [10] developed an activity monitoring system specifically to monitor elderly behavior. The system involved continuous tracking and studying of elderly behavior to interpret any associated anomalies resulting from behavioral disorders or motor impairments. Suriani et al. [11] proposed a smartphone-based activity recognition framework that was neural network-driven. The authors used datasets to evaluate and discuss the performance characteristics of their approach. In [12], Mousavi et al. proposed a similar smartphone-based application for monitoring elderly behavior. The system functioned by using a trained Support Vector Machine (SVM) that could monitor the behavioral patterns of the elderly to detect falls with an accuracy of $96.33 \%$. Alarifi [13] developed a wearable sensor-driven fall detection system that involved six wearables being placed on specific locations of the user's body. These wearables collected multiple components of the pose, posture, and gesture-related data studied by a neural network to detect falls. Al-Okby et al. [14] developed a similar smart wearable device for fall detection.

The system developed by Idrees et al. [15] involved scanning QR codes for indoor location tracking. Several scannable QR codes were set up in the user's functional environment. The user's location was determined by the system based on the associated QR codes that the user scanned using their phone. An indoor localization system that used smart cameras and image processing principles was developed by Chaccour et al. [16]. The cameras were fixed at specific locations to cover the entire space where a person could move around in the simulated environment. The person moving around in the environment carried markers on their head, which were tracked by these smart cameras to detect the changes in the person's position. Sun et al. [17] used smart cameras to develop a methodology of subtracting a background in an indoor location. This subtraction of the background from the user's spatial data allowed to track the user's location in the given space. A sensor technology-driven indoor localization system was proposed by Desai et al. [18]. In this system, the difference in time arrival methodology was used while analyzing the video data from sensors to interpret the user's location. Rituerto et al. [19] used image processing and computer vision technologies to develop a system for indoor localization. Endo et 
al. [20] proposed an LSD-SLAM-based approach for tracking and locating a user in a given indoor space.

As shown in these works, the methodologies [10-14] that focused on human activity recognition and analysis cannot detect the user's indoor location. Simultaneously, the works [15-20] that focused on indoor localization did not focus on analyzing the multimodal components of human activities. The proposed methodology of Pervasive Activity Logging for Indoor Localization aims to address these challenges by being able to (1) analyze human activities in pervasive environments along with the associated characteristics of these activities in terms of the contextual, behavioral, spatial, and temporal information and (2) detect the indoor location of the user in the context of the diversified and dynamic range of user interactions with context parameters during these activities. This methodology of Pervasive Activity Logging for Indoor Localization is discussed in Section 3.

\section{PROPOSED APPROACH}

In this section, we discuss the associated steps for developing the Pervasive Activity Logging for Indoor Localization. There are nine steps, and they are as follows:

1) Data capture and collection

Consider an indoor space layout and track the indoor navigational paths associated with different ADLs to set up and deploy wireless and wearable sensors in a Big Data-driven IoT-based data collection system.

2) Data analysis to extract the specific goal-oriented parametric data of different activities

Set up a pervasive human activity recognition system that can study the Big Data associated with the goal, motive, and intent of the human subject and user interactions with physical objects during different activities, tracked by the data collection system within the given pervasive environment.

3) Activity analysis

Analyze the context-driven components of all the activities by decomposing events into a finer granularity of motions, tasks, and sub-tasks (as per the goal, motive, and intent) and label them into atomic activities, context attributes, core atomic activities, and core context attributes with their associated weights [21].

4) Behavioral and motion analysis

Study the associated behavioral characteristics in terms of postures, gestures, and movements by interpreting human behavior as a function of joint point characteristics obtained by skeletal tracking [22].

5) Interaction sequence interpretation and analysis

Map the various user interaction patterns for each activity to develop a knowledge base of all possible forms of user interactions associated with the given activity by considering the motions, tasks, and sub-tasks and including both false starts and context-driven direct or indirect distractions. Interpret the spatial and temporal characteristics of the activities to understand their interdependent and interrelated characteristics.

6) Activity spatial semantics analysis - mapping activities and motions to indoor locations
Study the semantic components of all user interactions associated with these activities to detect the associated 'activity-based zone' in which the user was present during each of these activities [23]. These 'zones' represent the confines of the areas in the spatial environment of the user where a specific activity is performed. For instance, the activity of cooking can be mapped to a cooking 'zone.' Here, the process of mapping [23] states that if a user is performing the activity of cooking at a given point of time, the user would not be present in any other 'zone' of the environment such as the bathroom 'zone.'

7) Develop an exhaustive activity and event 'log'

Develop a database or a 'log' consisting of all these characteristics of the activities performed in the different 'zones' local to the given IoT-based space.

8) Detect and catalog dynamic event data and its interpretation Develop and implement the adaptive nature of this database for any of these scenarios - (a) when a new activity that was not recognized by Step 2 is performed in the confines of the given IoTbased space; (b) any change or modifications with the behavioral characteristics associated with any of the activities which were not detected by Step 4 before; (c) any new patterns of false starts or context-driven direct or indirect distractions associated with the activities; and (d) changes in the associated temporal or spatial information originally detected by Step 5 . Here, by adaptive nature, we refer to the functionality of the database to update itself either by modifying existing data or by adding new data to the records based on whenever any updated or new activity-related information is available and tracked by the system.

9) Develop a machine learning system (MLS) and test for the efficacy of learned patterns

Split this dataset after performing the data preprocessing steps (Steps 3-8) into the training set and test set using 75\% data for training and $25 \%$ data for testing. Use machine learning and pattern recognition principles to set up a learning model that uses the described steps above to train a Random Forest-based classification approach to detect the user's indoor location during different activities. Set the number of Random Trees associated with this Random Forest model as 250 and the maximum depth of a tree as 25. Evaluate the machine learning model's performance on the test data using a confusion matrix that presents both the overall accuracy and class precision values. Here, the individual class precision values correspond to the precision by which the user can be detected in each of the multiple 'zones' in the given environment based on the user interactions, behavioral features, and the spatial and temporal components of the activities.

The implementation of these steps is explained below. Steps (1) through (6) require using a specific pervasive indoor space, say, a Smart Home environment, with well-defined 'zones' for various ADLs. We have set up a configuration of a living space to contain a cooking area, eating area, entertainment area, studying area, and resting area in our lab space [24]. We have obtained IRB approval from our institution to carry out experiments with human subjects within this space. However, due to the Covid-19 situation and the associated 'work from home' recommendations by various government sectors in the United States [25], we could not collect 


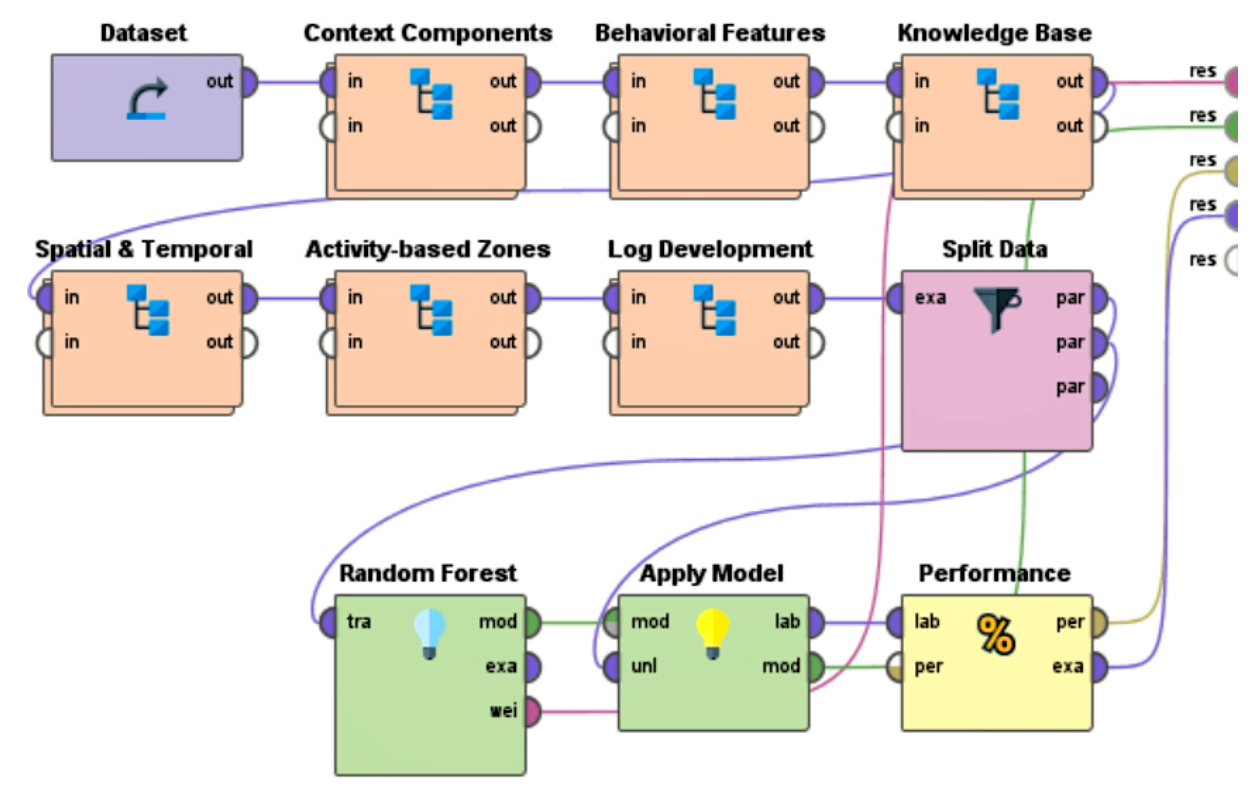

Figure 1: The RapidMiner "process" for development of the proposed approach for Pervasive Activity Logging for Indoor Localization

real-time data using this data collection framework [24]. So, we used a dataset developed by Ordóñez et al. [26]. This dataset was specifically chosen because its associated attributes were the same as the real-time data that our data collection framework could have collected. This dataset consists of multiple activities that were performed in an IoT-based pervasive environment. These activities include going to bed, preparing breakfast, taking a shower, leaving the house, storing groceries, getting a drink, preparing dinner, and getting a snack.

Steps (7) through (9) of this Pervasive Activity Logging for Indoor Localization focus on processing the data using machine learning and pattern recognition-based methodologies. We implemented all the steps associated with this methodology in RapidMiner [27] RapidMiner is a software application development tool that allows the development and implementation of various Big Data, Machine Learning, Artificial Intelligence, Internet of Things, and Information Retrieval-related algorithms. We used RapidMiner specifically for two reasons - (1) the associated functionalities of RapidMiner allow seamless development of an application that allows integration of various methodologies and advancements from Big Data, Machine Learning, Artificial Intelligence, Pattern Recognition, Internet of Things, and Information Retrieval; and (2) the characteristic features of RapidMiner allows development and customization of such an application that can communicate with other platforms and can be integrated with the same. We used the free version of RapidMiner Studio 9.8.001 on a Microsoft Windows 10 computer. Although the free version has a data processing limit of 10,000 rows, this did not affect any of our results, findings, or inferences, as the total number of rows in the dataset [26] was less than 10,000.

As we have used RapidMiner to develop this proposed methodology, we define two terminologies specific to RapidMiner here in a broad manner. These are "process" and "operator." In RapidMiner, an "operator" refers to one of the multiple building blocks of an application associated with specific functionalities that can be changed or modified either statically or dynamically, both by the user and by the application based on the specific need. In RapidMiner, certain "operators" are already developed in the software tool, which can be customized or updated. The tool also allows the development of new "operators" based on any specific need. A continuous, logical, and operational collection of "operators" linearly or hierarchically representing a working application with one or more output characteristics is referred to as a "process." The RapidMiner "process" that represents this methodology is shown in Figure 1.

The 'Dataset' operator was used to import the dataset [26] into RapidMiner. The 'Context Components' operator was developed and used to analyze the context-driven components of all the activities such as atomic activities, context attributes, core atomic activities, core context attributes as per their associated weights, as outlined in Step 3. The 'Behavioral Features' operator was developed and used to study the associated behavioral characteristics in terms of postures, gestures, and movements by interpreting human behavior as a function of joint point distance and joint point speeds, as discussed in Step 4. The 'Knowledge Base' operator was developed and used to map the various user interaction patterns for each activity to develop a knowledge base of all possible forms of user interactions associated with the given activity, including both false starts and context-driven direct or indirect distractions as outlined in Step 5. The 'Spatial \& Temporal' operator was developed and used to interpret the spatial and temporal characteristics of the activities to understand their interdependent and interrelated characteristics, as discussed in Step 5. The 'activity-based zones' operator was developed and used to study the semantic components 


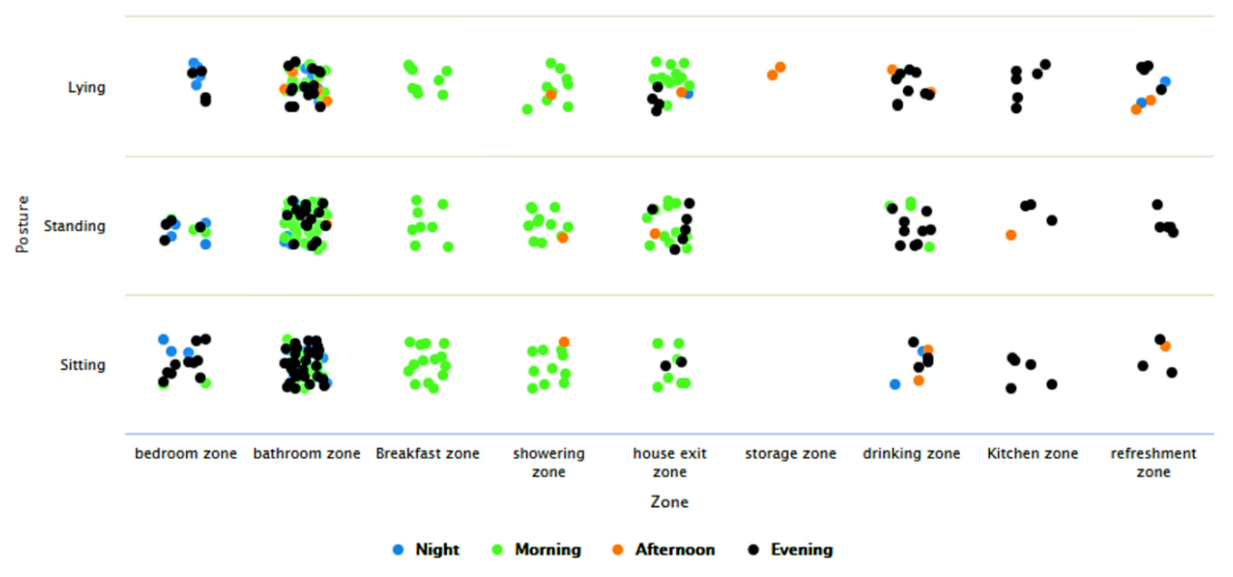

Figure 2: Result from the RapidMiner "process" that shows the different behavioral patterns that were tracked during different activities performed in each of these 'activity-based zones' during different times of the day

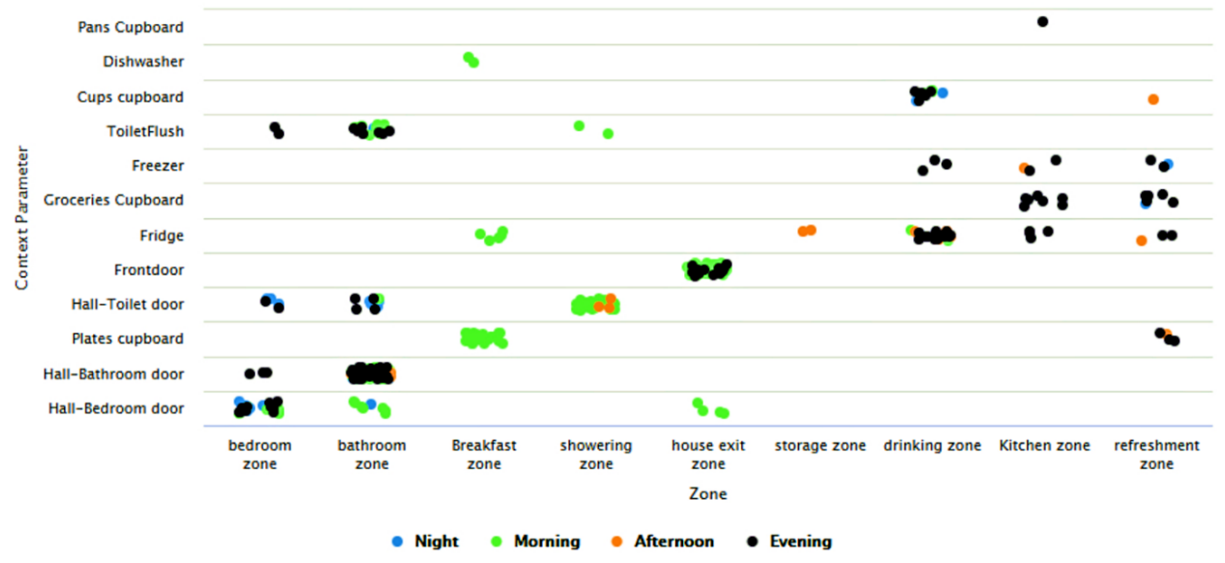

Figure 3: Result from the RapidMiner "process" that shows the distinct context parameters that characterize the user interactions tracked in each of these 'zones' during different time instants of the day

of all user interactions associated with these activities to detect the associated 'activity-based zone' in which the user was present during these activities, as outlined in Step 6. The 'Log Development' operator was developed and used to create an adaptive database or a ' log' that consists of all these characteristics of the activities performed in the different 'zones' local to the given IoT-based space, as discussed in Steps 7 and 8. The 'Split Data' operator was used to split the data into the training set and test set. $75 \%$ of the data was used for training, and the remaining $25 \%$ was used for testing. After that, we used the 'Random Forest' operator by customizing its features as discussed in Step 9 to set up a Random Forest-based learning model. The 'Apply Model' operator was used to apply this learning model to the test data. The 'Performance' operator was customized as outlined in Step 9 and connected to the 'Apply Model' operator to evaluate the performance characteristics of this approach. The overall performance accuracy of this RapidMiner process was found to be $83.02 \%$. Further discussions about the same are presented in Section 4.

\section{RESULTS AND DISCUSSIONS}

This section presents the results of developing the proposed Pervasive Activity Logging approach for Indoor Localization as per Steps 1-9 outlined in Section 3. The RapidMiner process for the development of the same is shown in Figure 1. Using this RapidMiner process, we first studied and analyzed the different behavioral patterns associated with different 'activity-based zones' in the given dataset. This relationship between behaviors, activities, and activitybased 'zones' along with the associated temporal information is shown in Figure 2, which is color-coded according to the different time instants of the day - morning, afternoon, evening, and night, during which these behaviors were tracked in each of these distinct 'activity-based zones.'

The associated distinct behavioral patterns that were found in this dataset included standing, sitting, and lying. The different activity-based zones were the bedroom 'zone', bathroom 'zone', breakfast 'zone', showering 'zone', house exit 'zone', storage 'zone', drinking 'zone', kitchen 'zone', and refreshment 'zone'. These 


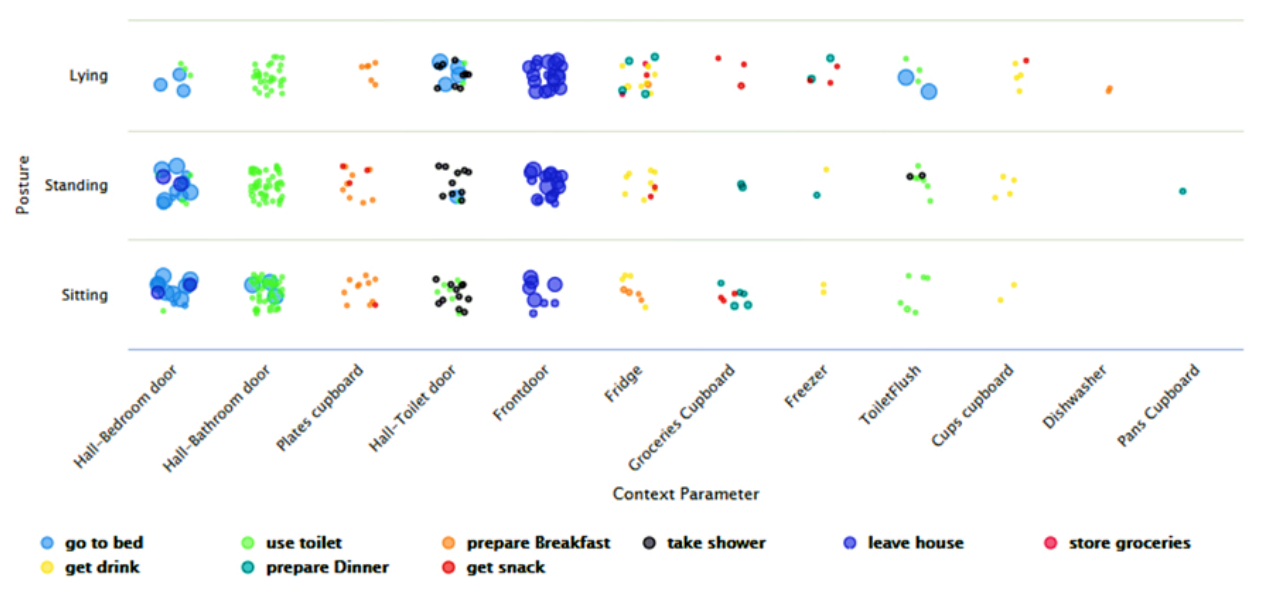

Figure 4: Result from the RapidMiner "process" that shows the interrelated and interdependent relationships between the postures and the context parameters with respect to the time duration spent by the user in each of these 'activity-based zones'

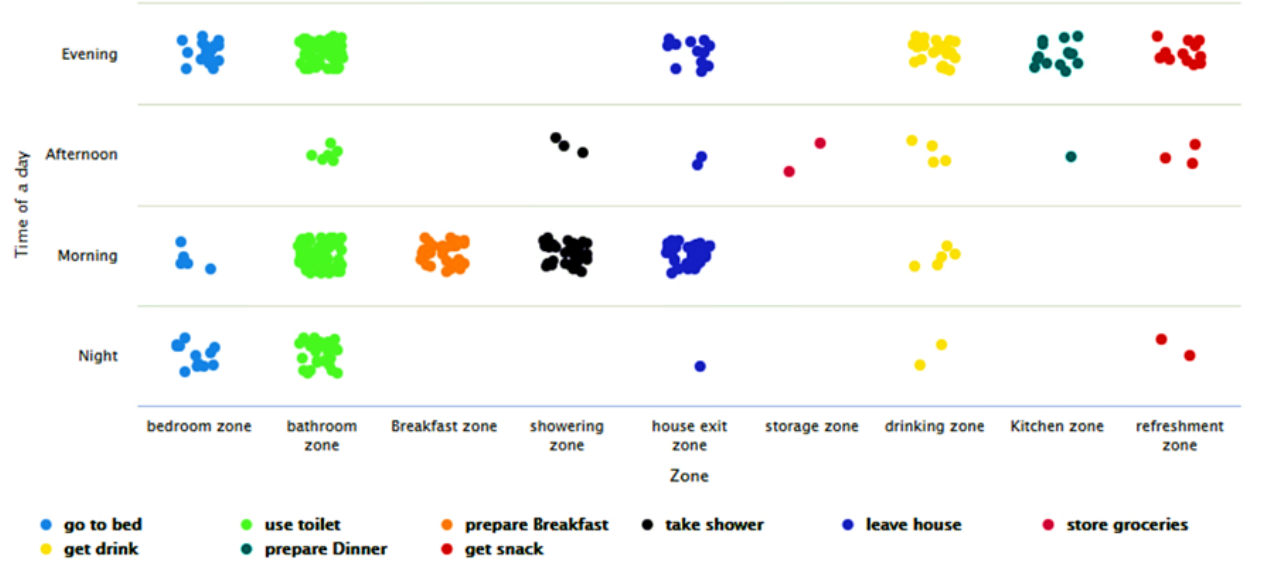

Figure 5: Result from the RapidMiner "process" that shows the distinct activities that took place in diverse ways in each of these 'activity-based zones' at different times instants of the day

'activity-based zones' were developed based on the associated user interaction features and behavioral characteristics associated with the different activities found in the dataset, as outlined in Step 6. In Figure 2, the $\mathrm{X}$-axis represents the distinct 'activity-based zones.' The Y-axis represents the associated distinct behavioral features tracked during different activities performed in each of these 'activity-based zones.' After that, we studied the context parameters that the user interacted with while performing the different activities local to each of these 'activity-based zones.' This interdependence of context parameters and 'zones' based on the related temporal characteristics is shown in Figure 3, where X-axis represents the different 'activity-based zones', and the Y-axis represents the various features or parameters of the environment that characterized the user interactions tracked in each of these 'zones'. These are color-coded according to the different time instants of the day morning, afternoon, evening, and night, during which these user interactions with the specific context parameters shown on the Y-axis took place.
Using the PAL approach's knowledge base, we analyzed the interrelated and interdependent relationships between the postures and the context parameters with respect to the time duration spent by the user in each of these 'activity-based zones'. This is represented in Figure 4. In Figure 4, the X-axis represents the context parameters. The Y-axis represents the distinct postures - standing, sitting, or lying associated with the user interactions with these context parameters. The plot is color-coded with respect to the different activities performed in each of these 'activity-based zones'. The size of each data point is based on the time duration for each activity. Here, a bigger data point represents greater time spent for that specific activity in that specific 'activity-based zone' at that specific instant and vice versa for a smaller data point. Then we studied the diverse ways in which the distinct activities took place in each of these 'activity-based zones' at different time instants by analyzing multimodal components of the user interactions and the associated behavioral patterns. These are shown in Figures 5 and 6 . 


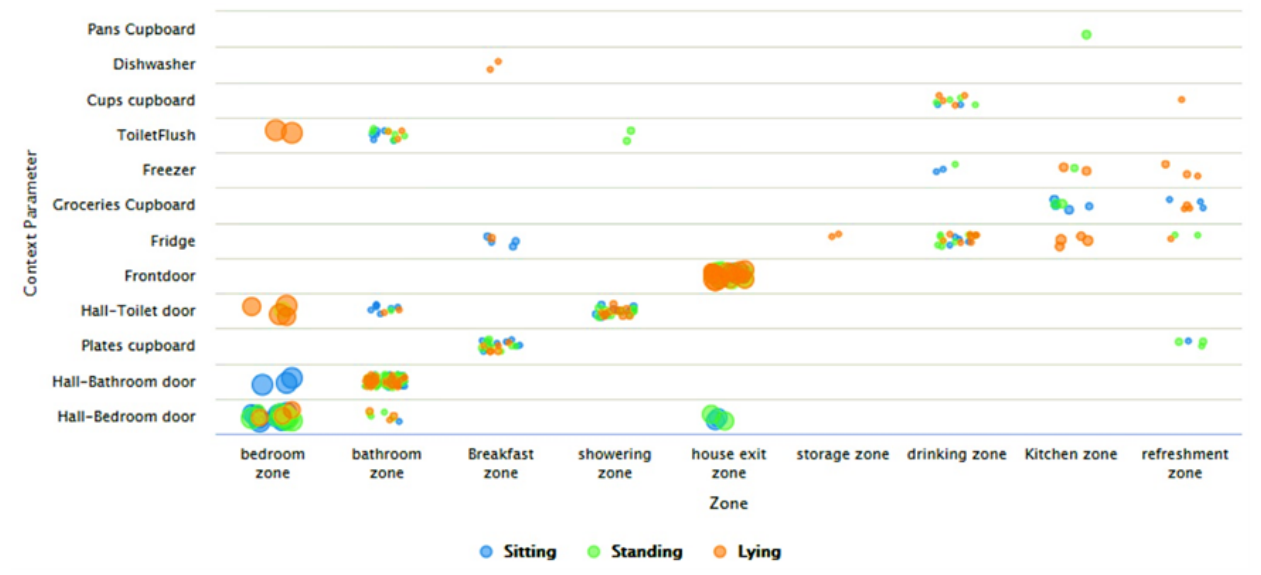

Figure 6: Result from the RapidMiner "process" that shows the relationship between context parameters local to each 'zone', the associated behavioral patterns, and the temporal information

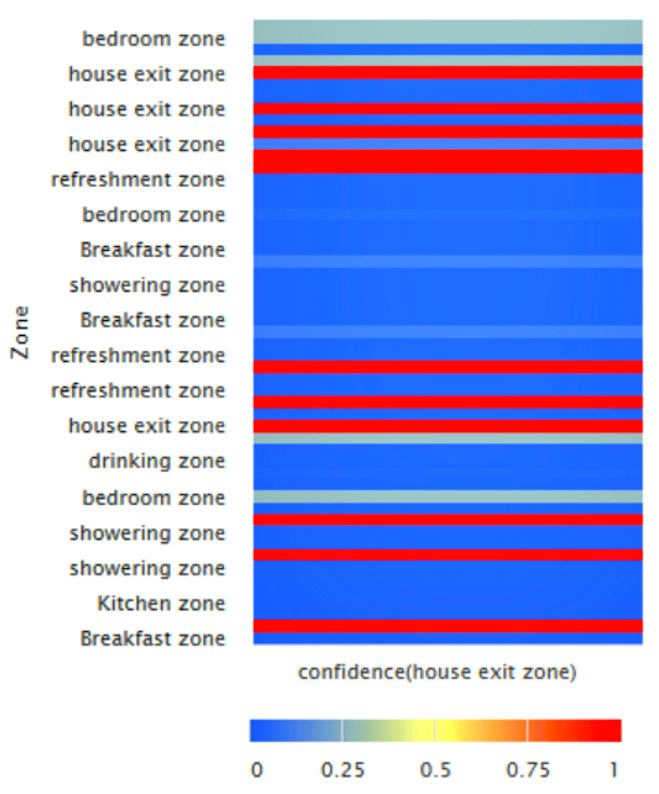

Figure 7: Result from the RapidMiner "process" (in the form of a heatmap) that shows the confidence of the learning model in detecting the location of the user in the house exit 'zone' during different activity instances

In Figure 5, the X-axis represents these 'activity-based zones,' and the Y-axis represents the time of the day. The different activities are represented in this figure using color-coding. These different activities included - going to bed, using the toilet, preparing breakfast, taking a shower, leaving the house, storing groceries, getting a drink, preparing dinner, and getting a snack. Figure 6 shows the relationship between context parameters local to each 'zone', the associated behavioral patterns, and the related temporal information. In this figure, the $\mathrm{X}$-axis represents the different 'zones'. The Y-axis represents the context parameters found in all these 'zones'. These

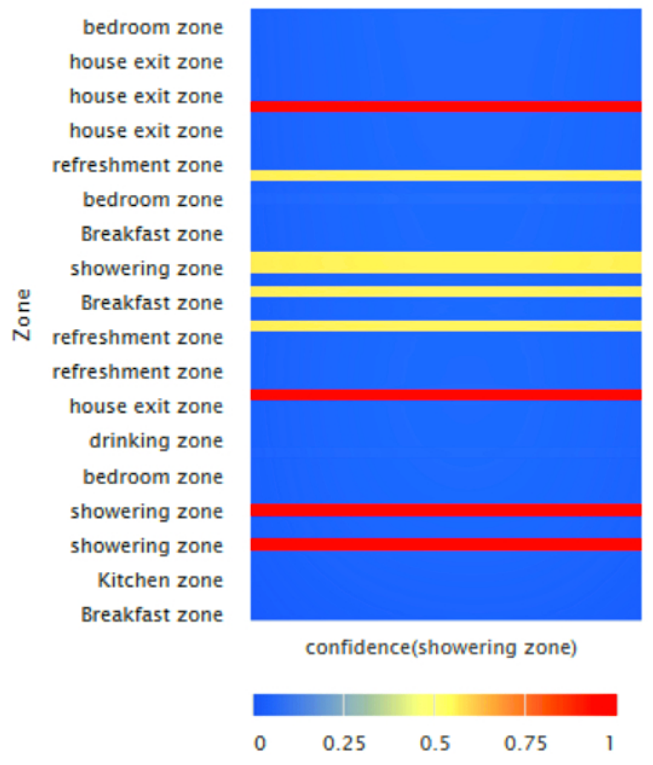

Figure 8: Result from the RapidMiner "process" (in the form of a heatmap) that shows the confidence of the learning model in detecting the location of the user in the showering 'zone' during different activity instances

data points are color-coded according to the associated behavioral patterns - sitting, standing, and lying. The size of each of these data points is according to the time the user spent for each of these activities, where a bigger data point indicates more time spent and a smaller data point indicates less time spent.

The developed Random Forest model worked by assigning a confidence value for each 'zone' every time the user performed an activity. Or in other words, this learning model assigned confidence values to the bedroom 'zone', bathroom 'zone', breakfast 'zone', showering 'zone', house exit 'zone', storage 'zone', drinking 'zone', 


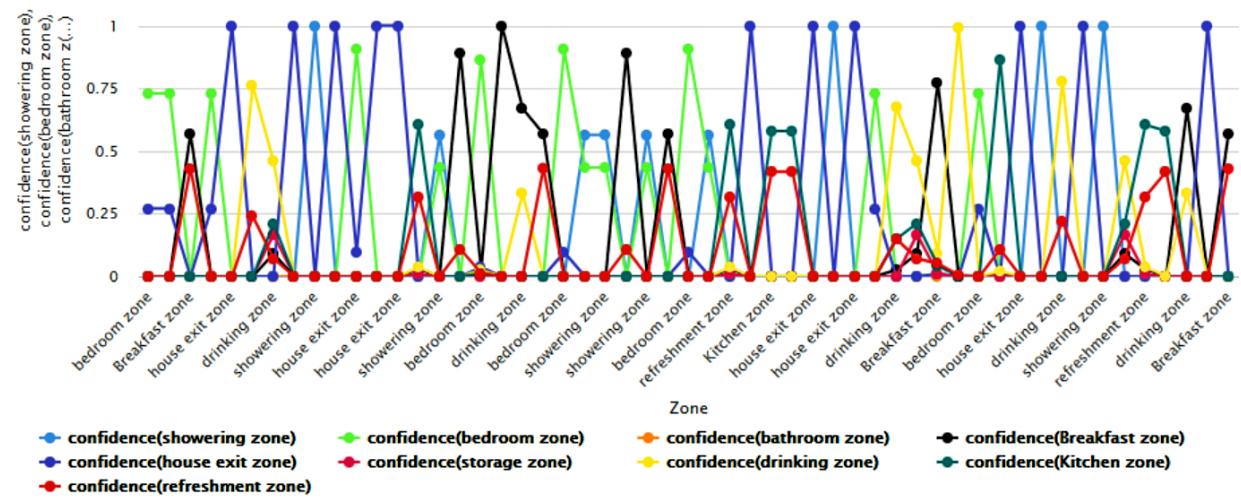

Figure 9: Result from the RapidMiner "process" that shows the Random Forest-based model's working where it assigned a confidence value to each 'activity-based zone' (only a few activities have been represented here).

kitchen 'zone', and refreshment 'zone' every time any activity was performed. This confidence value for each 'zone' represented the degree of confidence of this learning model in inferring that the user was in that 'zone' during the specific activity that was being performed. For any activity, the highest of these confidence values was considered by the learning model to deduce its inference of the location of the user in that 'zone'. Figures 7 and 8 show two heatmap representations of this learning model that shows the confidence of the same in deducing the indoor location of the user in the house exit 'zone' and in the showering 'zone', respectively, during different activity instances.

For lack of space, not all activity instances and the associated zones are represented in Figures 7 and 8 . In each of these figures, the Y-axis represents the different 'activity-based zones'. In Figure 7, the $\mathrm{X}$-axis represents the learning model's confidence value in inferring the user's location in the house exit 'zone' for all these activities performed in the different 'zones'. Similarly, in Figure 8, the Xaxis represents the learning model's confidence value in inferring the user's location in the showering 'zone' for all these activities performed in the different 'zones'. These confidence values are represented as per the color indicator shown in both these figures.

Figure 9 represents some typical activity instances from this dataset to discuss the working of the model in detecting the user's 'zone-based' indoor location. Here, the X-axis represents the actual locations of the user in different 'zones' at different time instants, and the Y-axis represents all the confidence values associated with the distinct 'activity-based zones' for each of these time instants. These confidence values were in the range of 0 to 1 for each such activity instance. For clarity of representation, activity instances where the confidence values associated with one or more 'zones' were very low, or in other words, close to 0 , have not been plotted in Figure 9. The performance characteristics of this machine learning model were studied by using a confusion matrix as outlined in Step 9 in Section 3. A confusion matrix [28] is a methodology for assessing and analyzing the output characteristics of a machine learning-based algorithm. Each row of the matrix represents the number of instances of a data label in the predicted class, while each column of the matrix represents the number of instances of a data label in the actual class. This matrix is sometimes inverted to study the rows as columns and the columns as rows. This methodology analyzes several performance metrics of the machine learning model being studied, including - overall accuracy, individual class precision values, recall, specificity, positive predictive values, negative predictive values, false-positive rates, false-negative rates, and F-1 scores. In our work, we evaluated the proposed learning approach based on the overall accuracy, the class precision, and the class recall values, which can be calculated as shown in equations 1), (2), and (3), respectively:

$$
\begin{gathered}
\text { Overall_Accuracy }=\frac{\text { True_P }+ \text { True_N }}{\text { True_P }+ \text { True_N }+ \text { False_P False_N }} \\
\text { Class_Precision }=\frac{\text { True_P }}{\text { True_P } P \text { False_P }} \\
\text { Class_Recall }=\frac{\text { True_P }}{\text { True_P }+ \text { False_N }}
\end{gathered}
$$

where:

Overall_Accuracy = overall accuracy of the machine learning model True_P $=$ true positive

True_N = true negative

Class_Precision $=$ sub class precision

The tabular view of the confusion matrix is shown in Figure 10. As shown in Figure 10, this indoor localization approach's overall performance accuracy was $83.02 \%$. The respective class precision values and class recall values for each of these 'zones' can be observed from the last column and the last row of Figure 10. These respective class precision values represent the accuracy of this learning model for detecting the user in each of these 'activity-based zones' - bedroom zone, bathroom zone, breakfast zone, showering zone, house exit zone, storage zone, drinking zone, kitchen zone, and refreshment zone. For instance, the class precision values for detecting the user in the bedroom 'zone' and breakfast 'zone' were $88.89 \%$ and $80.00 \%$, respectively. The associated class recall values were $88.89 \%$ and $100.00 \%$, respectively.

\section{CONCLUSIONS}

The rapidly increasing population of elderly people, associated with multiple challenges and limitations such as cognitive impairment, behavioral disorders, motor disabilities, weakened memory, and 


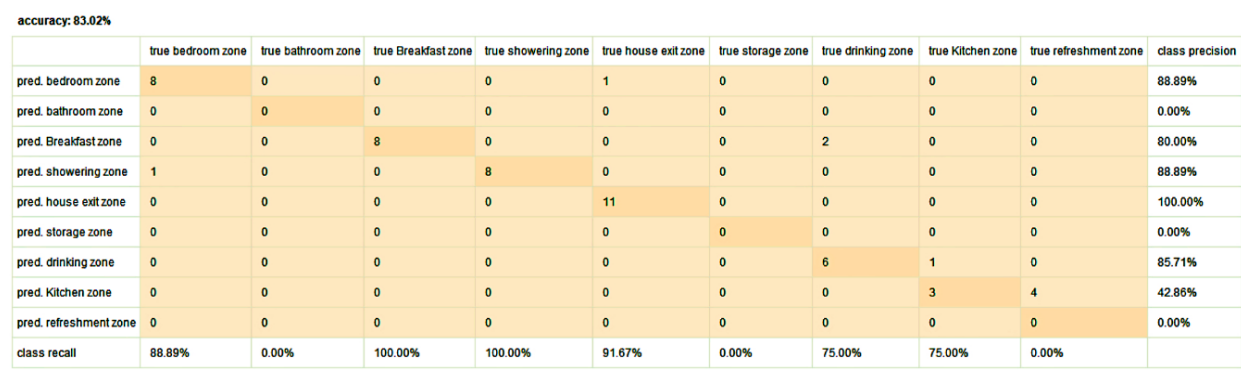

Figure 10: Performance characteristics in the form of a confusion matrix (tabular view) from the RapidMiner "process" for implementing the proposed Pervasive Activity Logging for Indoor Localization.

visual impairments, is posing a huge burden on the world economy due to their increasing and diversified needs. With the decreasing count of caregivers, it is the need of the hour for the futuristic Internet of Things (IoT)-based pervasive living environments, such as Smart Homes, to be able to sense, anticipate, adapt, predict, and respond to human behavior and their associated diverse needs. Such functionalities in Smart Homes would allow collecting activitycentered information from the Big Data of user interactions during activities of daily living (ADL) for interpretation of the interrelated and interdependent contextual, behavioral, spatial, and temporal characteristics during ADL. This would pave the way for creating user-centered smart and adaptive systems to address dynamic user needs as per varying user diversities, with a specific focus on addressing the needs of the elderly to contribute towards their independent living, healthy aging, and improved quality of life in the future of pervasive living environments.

Thus, this paper proposes and discusses a new computing methodology, Pervasive Activity Logging (PAL), that can take a holistic approach towards addressing these challenges. The development of this methodology involved integrating advancements from Pervasive Computing, Big Data, Information Retrieval, Internet of Things, and Human-Computer Interaction. There can be multiple applications of such a computing methodology, and we focus on one specific application - Indoor Localization, in this paper. Machine Learning and Pattern Recognition techniques and methodologies were used to develop and demonstrate this specific application of PAL. A Random Forest-based classifier was tested on a dataset that consisted of 9 different activities performed within the premises of an IoT-based pervasive environment. It achieved an overall performance accuracy of $83.02 \%$ for detecting the user's indoor location in a specific 'activity-based zone'. According to the authors' best knowledge, no similar work has been done in this field thus far. The results presented and discussed uphold the immense relevance and importance of this approach for the future of IoT-based pervasive living environments, such as Smart Homes. The results also demonstrate the potential of this PAL approach for addressing the multiple global societal and economic challenges posed due to the diverse aging population and their needs.

The work presented in this paper and the associated discussions also show that by using recent advancements from multiple disciplines, the computing methodology of Pervasive Activity Logging holds the potential for supporting the development of a range of other applications in the future of IoT-based pervasive environments such as - (1) user-centered behavior interventions during ADL; (2) development of association rules between different activities for the development of user-specific task recommendation schemas; (3) personalized activity recommendation systems; (4) multi-user task recommendation systems for augmenting group performance as well as individual user performances. Future work would involve using and expanding the computing methodology of Pervasive Activity Logging into developing these systems and applications. We also plan on investigating methodologies such as hyperparameter tuning and cross-validation as well as developing and implementing a deep learning-based approach to improve the performance accuracy of the model for detecting the user in a specific 'zone' at a given point of time by incorporating the associated temporal information and other characteristics of user interactions.

\section{CONFLICT OF INTEREST}

The authors declare that there is no conflict of interest regarding the publication of this paper.

\section{ACKNOWLEDGMENTS}

This work was supported by a Grant in Aid of Research from the National Academy of Sciences, administered by Sigma Xi, The Scientific Research Honor Society. The authors would like the thank the National Academy of Sciences and Sigma Xi, The Scientific Research Honor Society for this support.

\section{REFERENCES}

[1] "Decade of Healthy Ageing," Who.int. [Online]. Available: https://www.who.int/ initiatives/decade-of-healthy-ageing. [Accessed: 12-Mar-2021]

[2] H. Arslantas, F. Adana, F. Abacigil Ergin, D. Kayar, and G. Acar, "Loneliness in elderly people, associated factors and its correlation with quality of life: A field study from western turkey," Iran. F. Public Health, vol. 44, no. 1, pp. 43-50, 2015.

[3] "Dementia," Who.int. [Online]. Available: https://www.who.int/news-room/factsheets/detail/dementia. [Accessed: 12-Mar-2021].

[4] V. R. Strang, P. M. Koop, S. Dupuis-Blanchard, M. Nordstrom, and B. Thompson, "Family caregivers and transition to long-term care," Clin. Nurs. Res., vol. 15, no. 1, pp. 27-45, 2006.

[5] M. Haymaker, "Care networking, care anywhere, care customization," Cisco.com, 22-Oct-2013. [Online]. Available: https://blogs.cisco.com/healthcare/ care-networking-care-anywhere-care-customization. [Accessed: 12-Mar-2021].

[6] M. Blum, A. Pentland, and G. Troster, "InSense: Interest-based life logging," IEEE multimed., vol. 13, no. 4, pp. 40-48, 2006.

[7] N. Thakur, "Framework for a context aware adaptive intelligent assistant for activities of daily living," Master's Thesis, University of Cincinnati, 2019.

[8] C. Langlois, S. Tiku, and S. Pasricha, "Indoor localization with smartphones: Harnessing the sensor suite in your pocket," IEEE consum. electron. Mag., vol. 6, 
no. 4 , pp. $70-80,2017$

[9] C. M. Ranieri, S. MacLeod, M. Dragone, P. A. Vargas, and R. A. F. Romero, "Activity recognition for Ambient Assisted Living with videos, inertial units and ambient sensors," Sensors (Basel), vol. 21, no. 3, p. 768, 2021.

[10] L. G. Fahad and S. F. Tahir, "Activity recognition and anomaly detection in smart homes," Neurocomputing, vol. 423, pp. 362-372, 2021.

[11] N. S. Suriani and F.' atyka N. Rashid, "Smartphone sensor accelerometer data for human activity recognition using spiking neural network.", International Journal of Machine Learning and Computing, Vol. 11, No. 4, July 2021.

[12] S. A. Mousavi, F. Heidari, E. Tahami, and M. Azarnoosh, "Fall detection system via smart phone and send people location," in 2020 28th European Signal Processing Conference (EUSIPCO), 2021, pp. 1605-1607.

[13] A. Alarifi and A. Alwadain, "Killer heuristic optimized convolution neural network-based fall detection with wearable IoT sensor devices," Measurement (Lond.), vol. 167, no. 108258, p. 108258, 2021

[14] M. F. R. Al-Okby and S. S. Al-Barrak, "New approach for fall detection system using embedded technology," in 2020 IEEE 24th International Conference on Intelligent Engineering Systems (INES), 2020, pp. 209-214.

[15] A. Idrees, Z. Iqbal, and M. Ishfaq, "An efficient indoor navigation technique to find optimal route for blinds using QR codes," in 2015 IEEE 10th Conference on Industrial Electronics and Applications (ICIEA), 2015, pp. 690-695.

[16] K. Chaccour and G. Badr, "Computer vision guidance system for indoor navigation of visually impaired people," in 2016 IEEE 8th International Conference on Intelligent Systems (IS), 2016, pp. 449-454.

[17] Y. Sun, K. Zhao, J. Wang, W. Li, G. Bai, and N. Zhang, "Device-free human localization using panoramic camera and indoor map," in 2016 IEEE International Conference on Consumer Electronics-China (ICCE-China), 2016, pp. 1-5

[18] P. Desai and K. S. Rattan, "Indoor localization and surveillance using wireless sensor network and Pan/Tilt camera," in Proceedings of the IEEE 2009 National Aerospace \& Electronics Conference (NAECON), 2009, pp. 1-6.

[19] A. Rituerto, G. Fusco, and J. M. Coughlan, "Towards a sign-based indoor navigation system for people with visual impairments," ASSETS, vol. 2016, pp. 287-288,
2016.

[20] Y. Endo, K. Sato, A. Yamashita, and K. Matsubayashi, "Indoor positioning and obstacle detection for visually impaired navigation system based on LSD-SLAM," in 2017 International Conference on Biometrics and Kansei Engineering (ICBAKE), 2017, pp. $158-162$.

[21] S. Saguna, A. Zaslavsky, and D. Chakraborty, "Complex activity recognition using context-driven activity theory and activity signatures," ACM Trans. Comput. Hum. Interact., vol. 20 , no. 6 , pp. 1-34, 2013

[22] N. Thakur and C. Y. Han, "Towards a language for defining human behavior for complex activities," in Human Interaction, Emerging Technologies and Future Applications III, Cham: Springer International Publishing, 2021, pp. 309-315.

[23] N. Thakur and C. Y. Han, "An ambient intelligence-based human behavior monitoring framework for ubiquitous environments," Information (Basel), vol. 12, no. 2, p. 81, 2021.

[24] S. Chakraborty, C. Y. Han, X. Zhou, and W. G. Wee, "A context driven human activity recognition framework," in Proceedings of the 2016 International Conference on Health Informatics and Medical Systems, Monte Carlo Resort, Las Vegas, NV, USA, 25-28 July 2016; pp. 96-102.

[25] B. Axelrod, "Ohio Gov. Mike DeWine asks employers to continue working remotely amid COVID-19," WKYC, 30-Nov-2020. [Online]. Available: https://www.wkyc.com/article/news/health/coronavirus/dewine-wokingremotely-covid-19/95-54447569-e757-4eac-bbc3-4bab4652b764. [Accessed: 12-Mar-2021].

[26] F. J. Ordóñez, P. de Toledo, and A. Sanchis, "Activity recognition using hybrid generative/discriminative models on home environments using binary sensors," Sensors (Basel), vol. 13, no. 5, pp. 5460-5477, 2013.

[27] I. Mierswa, M. Wurst, R. Klinkenberg, M. Scholz, and T. Euler, "YALE: Rapid prototyping for complex data mining tasks," in Proceedings of the 12th ACM SIGKDD international conference on Knowledge discovery and data mining - KDD '06, 2006.

[28] Confusion Matrix. Available online: https://en.wikipedia.org/wiki/Confusion matrix (accessed on 1 February 2021) 